\title{
Homotopy types of one-dimensional Peano continua
}

\author{
by
}

\author{
Katsuya Eda (Tokyo)
}

\begin{abstract}
Let $X$ and $Y$ be one-dimensional Peano continua. If the fundamental groups of $X$ and $Y$ are isomorphic, then $X$ and $Y$ are homotopy equivalent. Every homomorphism from the fundamental group of $X$ to that of $Y$ is a composition of a homomorphism induced from a continuous map and a base point change isomorphism.
\end{abstract}

1. Introduction and definitions. In this paper we prove:

Theorem 1.1. Let $X$ and $Y$ be one-dimensional Peano continua. If the fundamental groups of $X$ and $Y$ are isomorphic, then $X$ and $Y$ are homotopy equivalent.

Theorem 1.2. Let $X$ be a one-dimensional Peano continuum, $Y$ a onedimensional metric space and $x \in X$ and $y \in Y$. For each homomorphism $h: \pi_{1}(X, x) \rightarrow \pi_{1}(Y, y)$ there exists a continuous map $f: X \rightarrow Y$ and a path $q$ from $f(x)$ to $y$ such that $h=\varphi_{q} \circ f_{*}$, where $\varphi_{q}$ is the base point change isomorphism.

Corollary 1.3. Let $X$ and $Y$ be one-dimensional Peano continua and $f: X \rightarrow Y$ a continuous map. If $f$ induces an isomorphism between the fundamental groups of $X$ and $Y$, then $f$ is a homotopy equivalence between $X$ and $Y$.

It seems that the first theorem was conjectured in the middle 1990's; the author heard of it from $\mathrm{G}$. Conner. If the spaces $X$ and $Y$ are locally simply connected in addition to the conditions in Theorems 1.1 and 1.2, then $X$ and $Y$ are homotopy equivalent to finite graphs and their fundamental groups are free groups and consequently the conclusions of Theorems 1.1 and 1.2 are obvious. But in general they are rather nontrivial. On the other hand, if $X$ and $Y$ are not locally simply connected at any point, the isomorphism $h$

2010 Mathematics Subject Classification: Primary 55P10, 55P15; Secondary 55Q20, 55Q70, 54E45, 20F34.

Key words and phrases: fundamental group, one-dimensional, Peano continuum. 
between the fundamental groups of $X$ and $Y$ induces a homeomorphism $\tilde{h}$ between $X$ and $Y$ [8], which is not so trivial. Hearing Theorem 1.1] U. Karimov asked the author whether Corollary 1.3 holds and this is an affirmative answer to his question. Since our proofs extend those in [8] some of whose notions are uncommon, we restate a few definitions.

For $a \leq b$, a continuous map $f:[a, b] \rightarrow X$ is called a path from $f(a)$ to $f(b)$. The points $f(a)$ and $f(b)$ are called the initial point and the terminal point of $f$ respectively. When $a=b$, the path $f$ is said to be degenerate. A loop $f$ is a path with $f(a)=f(b)$. For a path $f:[a, b] \rightarrow X, f^{-}$denotes a path such that $f^{-}(s)=f(a+b-s)$ for $a \leq s \leq b$. Two paths $f$ : $[a, b] \rightarrow X, g:[c, d] \rightarrow X$ are equivalent, denoted by $f \cong g$, if there exists a homeomorphism $\varphi:[a, b] \rightarrow[c, d]$ such that $\varphi(a)=c, \varphi(b)=d$ and $f=g \circ \varphi$. Two paths $f:[a, b] \rightarrow X$ and $g:[c, d] \rightarrow X$ are homotopic, denoted by $f \sim g$, if there exists a continuous map $H$ whose domain is the quadrangle in the plane with the vertices $(a, 0),(b, 0),(c, 1)$ and $(d, 1)$ such that

$$
\left\{\begin{array}{l}
H(s, 0)=f(s) \quad \text { for } a \leq s \leq b, \\
H(s, 1)=g(s) \quad \text { for } c \leq s \leq d, \\
H((1-t) a+t c, t)=f(a)=g(c) \quad \text { for } 0 \leq t \leq 1, \\
H((1-t) b+t d, t)=f(b)=g(d) \quad \text { for } 0 \leq t \leq 1 .
\end{array}\right.
$$

The homotopy class containing a path $f$ is denoted by $[f]$. The homotopy defined above is usually called a "homotopy relative to end points". We drop the phrase "relative to end points" for simplicity.

A path $f:[a, b] \rightarrow X$ is reduced if no subloop of $f$ is null-homotopic, that is, for each pair $u<v$ with $f(u)=f(v), f \uparrow[u, v]$ is not null-homotopic. Note that a constant map is reduced if and only if it is degenerate. For paths $f:[a, b] \rightarrow X$ and $g:[c, d] \rightarrow X$ with $f(b)=g(c), f g$ denotes the concatenation of $f$ and $g$, that is, a path from $[a, b+d-c]$ to $X$ such that $f g(s)=f(s)$ for $a \leq s \leq b$ and $f g(s)=g(s-b+c)$ for $b \leq s \leq b+d-c$. A loop $f$ is cyclically reduced if $f f$ is reduced. An arc is a subspace of $X$ which is homeomorphic to the unit interval $[0,1]$. The Hawaiian earring is the plane continuum $\mathbb{H}=\bigcup_{n=1}^{\infty}\left\{(x, y):(x-1 / n)^{2}+y^{2}=1 / n^{2}\right\}$ and $o$ denotes the origin $(0,0)$. Each simple closed curve of the Hawaiian earring is parametrized as follows:

$$
\mathbf{e}_{n}(t)=((1+\cos (\pi+2 \pi t)) / n, \sin (\pi+2 \pi t) / n) \quad \text { for } 1 \leq n<\omega, 0 \leq t \leq 1 .
$$

Let $O^{X}$ consist of all elements $x \in X$ such that $X$ is locally simply connected at $x$. If $X$ is locally path-connected, then $O^{X}$ is an open subset of $X$. We define $X^{w}=X \backslash O^{X}$. For a homomorphism $h: \pi_{1}(X, x) \rightarrow G$, let $X_{h}^{w}$ be the set of all points $x_{0} \in X$ such that, for each neighborhood $U$ of $x_{0}$, there exists a loop $f$ in $U$ such that $h\left(\varphi_{g}([f])\right) \neq e$ for some path 
$g$ from $x_{0}$ to $x$. Let $O_{h}^{X}=X \backslash X_{h}^{w}$. We remark that in this definition the choice of a path $g$ does not affect anything. When $h$ is injective, we have $X_{h}^{w}=X^{w}$. We also remark that $X_{h}^{w}$ is closed and $O_{h}^{X}$ is open for a locally path-connected space $X$.

2. Reduction of one-dimensional Peano continua. A subset $A$ of a space $X$ is called an open arc if $A$ is open in $X$ and is homeomorphic to the open interval $(0,1)$. An open arc in a Peano continuum has at least one and at most two end points; we denote them by $A^{0}$ and $A^{1}$. We remark that $A^{0}=A^{1}$ may happen.

The next theorem has been proved in the master thesis of M. Meilstrup [15]. Since our proof will be modified to prove Theorem 1.2 , we prove this precisely.

Theorem 2.1 (M. Meilstrup [15]). Every one-dimensional Peano continuum is homotopy equivalent to a one-dimensional Peano continuum $X$ such that $X$ is a finite connected graph or $O^{X}$ is an at most countable union of open arcs the end points of which belong to $X^{w}$.

Our proof is a modification of the proof of [ 8 , Theorem 1.2], particularly that of the implication $(3) \Rightarrow(2)$, and hence we recommend the reader to review [8, Section 4] before proceeding.

A metric space $(X, \rho)$ is uniformly locally connected if, for every $\varepsilon>0$, there exists $\delta>0$ such that if $\rho(x, y)<\delta$ then $x$ and $y$ are contained in a connected open set of diameter less than $\varepsilon$. We refer the reader to [14, Section 2.1.1] for the notion of brick partiton and facts around it. An important fact is: if $O$ is a uniformly locally connected, connected open set in a Peano continuum, then $\bar{O}$ is also a Peano continuum.

A partition $\mathcal{P}$ of a space is a pairwise disjoint family of finitely many connected open sets such that $\bigcup \mathcal{P}$ is dense. A partition $\mathcal{P}$ is of order 2 if $\overline{P_{1}} \cap \overline{P_{2}} \cap \overline{P_{3}}=\emptyset$ for distinct $P_{1}, P_{2}, P_{3} \in \mathcal{P}$. A partition $\mathcal{P}$ is a brick partition if $\mathcal{P}$ consists of regular open sets and $\operatorname{int}(\bar{P} \cup \bar{Q})$ is uniformly locally connected for each $P, Q \in \mathcal{P}$. Consequently, each element of $\mathcal{P}$ is uniformly locally connected.

For a subset $S$ of $X$, the diameter of $S$ is denoted by $\operatorname{diam}(S)$, i.e. $\operatorname{diam}(S)=\sup \{\rho(x, y): x, y \in S\}$ and $\operatorname{Mesh}(\mathcal{P})=\max \{\operatorname{diam}(P): P \in \mathcal{P}\}$. Since our construction is based on the proof of [14, Theorem 2.9], we state that result in a form suitable for our case.

Proposition 2.2 ([14, Theorem 2.9]). Let $X$ be a one-dimensional Peano continuum, $K$ a 0 -dimensional closed subset of $X$ and $x_{0}, x_{1}, \ldots, x_{n} \in X$. Then, for every $\varepsilon>0$, there exists a brick partition $\mathcal{P}$ of $X$ of order 2 such that 
(1) $K \subseteq \bigcup \mathcal{P}$;

(2) if $x_{i} \neq x_{j}$, then there are distinct $P_{i}, P_{j} \in \mathcal{P}$ such that $x_{i} \in P_{i}$ and $x_{j} \in P_{j}$

(3) $\operatorname{Mesh}(\mathcal{P})<\varepsilon$;

(4) the boundary of each member of $\mathcal{P}$ is 0-dimensional.

Proof of Theorem 2.1. Let $X$ be a one-dimensional Peano continuum. If $X^{w}=\emptyset$, then the argument below shows that $X$ is homotopy equivalent to a finite connected graph. We therefore assume that $X^{w} \neq \emptyset$.

Our first goal is to construct dendrites $D_{n}$ and open $\operatorname{arcs} A_{i}$ so that

- $D_{n}$ is a dendrite such that $D_{n} \cap X^{w}=\left\{x_{n}\right\}, D_{n} \backslash\left\{x_{n}\right\} \subseteq O^{X}$ and $\partial D_{n} \subseteq \bigcup_{i} \partial A_{i} \cup\left\{x_{n}\right\}$

- $A_{i} \subseteq O^{X}$;

- $D_{m} \cap D_{n} \subseteq X^{w}$ for $m \neq n$ and $A_{i} \cap A_{j}=\emptyset$ for $i \neq j$;

- $X^{w} \cup \bigcup_{n} D_{n} \cup \bigcup_{i} A_{i}$ is a strong deformation retract of $X$;

- $\lim _{n \rightarrow \infty} \operatorname{diam}\left(D_{n}\right)=0$ and $\lim _{i \rightarrow \infty} \operatorname{diam}\left(A_{i}\right)=0$.

For this we construct brick partitions $\mathcal{P}_{m}$, open $\operatorname{arcs} A_{i}$, parts of dendrites $D_{m n}$, and points $y_{m n}$ by induction.

In the 0th step we let $\mathcal{P}_{0}=\{X\}$, but we do not define $A_{i}$ and so on. After the $m$ th step, we have finitely many points $y_{m n}$ on the boundary of $\bigcup\left\{\bar{P}: P \in \mathcal{P}_{m}, \bar{P} \cap X^{w} \neq \emptyset\right\}$. First we work in each $\bar{P}$ for $P \in \mathcal{P}_{m}$. Applying Proposition 2.2 to the 0 -dimensional closed set $\partial P$ and points $y_{m n}$ in $\partial P$, we have a brick partition $\mathcal{P}_{P}$ of $\bar{P}$ satisfying

- $\mathcal{P}_{P}$ is of order 2 and $\operatorname{Mesh}\left(\mathcal{P}_{P}\right)<1 /(m+1)$;

- if $\bar{Q} \cap X^{w}=\emptyset$ for $Q \in \mathcal{P}_{P}$, then $\bar{Q}$ is simply connected;

- if $\bar{Q} \cap X^{w}=\emptyset$ for $Q \in \mathcal{P}_{P}$, then $\bar{Q} \cap \overline{Q^{\prime}}$ is at most one point for $Q^{\prime} \in \mathcal{P}_{P}$ with $Q^{\prime} \neq Q$.

- If $y_{m n} \in \bar{P}$, then there exists $Q \in \mathcal{P}_{P}$ such that $y_{m n} \in Q$ and $\bar{Q} \cap X^{w}$ $=\emptyset$.

Next let $\mathcal{P}_{m+1}$ be the family

$$
\left\{Q \backslash \partial P \mid Q \in \mathcal{P}_{P}, P \in \mathcal{P}_{m}, \bar{P} \cap X^{w} \neq \emptyset\right\} .
$$

Since $\partial P$ does not separate any nonempty connected open set in $\bar{P}$ [14, Proposition 2.5], $\mathcal{P}_{m+1}$ is a partition of $\bigcup\left\{\bar{P}: P \in \mathcal{P}_{m}, \bar{P} \cap X^{w} \neq \emptyset\right\}$ and also a brick partition of it. Since $\partial P \subseteq \bigcup \mathcal{P}_{P}, \mathcal{P}_{m+1}$ is of order 2 . Hence $\mathcal{P}_{m+1}$ is a brick partition of $\bigcup\left\{\bar{P}: P \in \mathcal{P}_{m}, \bar{P} \cap X^{w} \neq \emptyset\right\}$ which satisfies the following:

(1) $\mathcal{P}_{m+1}$ is of order 2 and $\operatorname{Mesh}\left(\mathcal{P}_{m+1}\right)<1 /(m+1)$;

(2) $\mathcal{P}_{m+1}$ refines the restriction of $\mathcal{P}_{m}$ to $\bigcup\left\{\bar{P}: P \in \mathcal{P}_{m}, \bar{P} \cap X^{w} \neq \emptyset\right\}$;

(3) if $\bar{Q} \cap X^{w}=\emptyset$ for $Q \in \mathcal{P}_{m+1}$, then $\bar{Q}$ is simply connected; 
(4) if $\bar{Q} \cap X^{w}=\emptyset$ for $Q \in \mathcal{P}_{m+1}$, then $\bar{Q} \cap \overline{Q^{\prime}}$ is at most one point for $Q^{\prime} \in \mathcal{P}_{m+1}$ with $Q^{\prime} \neq Q$.

(5) If $y_{m n} \in \bar{P}$ for $P \in \mathcal{P}_{m}$ with $\bar{P} \cap X^{w} \neq \emptyset$, then there exists $Q \in \mathcal{P}_{m+1}$ such that $Q \subseteq P, y_{m n} \in \bar{Q}$ and $\bar{Q} \cap X^{w}=\emptyset$.

For each $Q \in \mathcal{P}_{m+1}$ with $\bar{Q} \cap X^{w}=\emptyset, \partial Q$ is finite and so we connect these points by arcs and have a finite tree $T_{Q}$ which is a strong deformation retract of $\bar{Q}$ in $\bar{Q}$, since $\bar{Q}$ is a uniquely arcwise connected Peano continuum. Then $\bigcup\left\{T_{Q}: Q \in \mathcal{P}_{m+1}, \bar{Q} \cap X^{w}=\emptyset\right\}=G_{m+1}$ is a finite graph, where we consider branching points in $T_{Q}$ and points in $\partial Q$ as vertices. Then $G_{m+1} \cap \bigcup\left\{\bar{Q}: Q \in \mathcal{P}_{m+1}, \bar{Q} \cap X^{w} \neq \emptyset\right\}$ is a finite set, which we enumerate without repetition as $y_{m+1 n}$.

For $y_{m l}$ we have a unique $P \in \mathcal{P}_{m}$ such that $y_{m l} \in \bar{P}$ and $\bar{P} \cap X^{w} \neq \emptyset$. According to (5) in the construction of $\mathcal{P}_{m+1}$, every point $y_{m l}$ is connected to at least one point $y_{m+1 n}$ by an arc $A$ so that $y_{m+1 n} \in \bar{Q}$ for $Q \in \mathcal{P}_{m+1}$ with $Q \subseteq P$ and $\bar{Q} \cap X^{w} \neq \emptyset$, and $A \backslash\left\{y_{m l}\right\} \subseteq P$. By working in each $P$ we have a tree $T_{n}^{P} \subseteq G_{m+1}$ so that $y_{m l}$ are connected to $y_{m+1 n}$ by $T_{n}^{P}$. Accordingly, if $y_{m+1 n}$ is connected to some $y_{m l}$, then $y_{m+1 n}$ belongs to a unique $P \in \mathcal{P}_{m}$. Therefore, there exists at most one $P$ such that a tree $T_{n}^{P}$ is connected to each $y_{m+1 n}$; we then let $T_{n}$ be $T_{n}^{P}$. When no $T_{n}^{P}$ is connected to $y_{m+1 n}$ we let $T_{n}$ be the singleton of $y_{m+1 n}$. In such a case $y_{m+1 n}$ may belong to $\bar{P} \cap \overline{P^{\prime}}$ for distinct $P, P^{\prime} \in \mathcal{P}_{m}$. But there exists a unique $P \in \mathcal{P}_{m}$ such that $y_{m+1 n} \in \bar{Q}$ for $Q \in \mathcal{P}_{m+1}$ with $Q \subseteq P$ and $\bar{Q} \cap X^{w} \neq \emptyset$. We work in the unique $P \in \mathcal{P}_{m}$ for such $T_{n}$ in the following procedure. We remark that the $T_{n}$ s are pairwise disjoint.

Vertices in $G_{m+1}$ which are in $\bar{P}$ may not belong to any $T_{n}$. Next we expand $T_{n}$ s in $G_{m+1}$ so that every vertex of $G_{m+1}$ belongs to one of the extensions of the trees $T_{n}$. We want to control the sizes of the expanded trees and so we work in each $\bar{P}$. By induction on $n$ we construct a maximal tree $T_{n}^{*}$ in $G_{m+1} \cap \bar{P}$ or $G_{m+1} \cap\left(\overline{P_{0}} \cup \overline{P_{1}}\right)$ such that $T_{n} \subseteq T_{n}^{*}$, but $T_{n}^{*} \cap \bigcup_{k<n} T_{k}^{*}=\emptyset$ and $T_{n}^{*} \cap \bigcup_{k>n} T_{k}=\emptyset$. Inductively, we assume that if $D_{m l}$ has been defined for $y_{m l}$ then $D_{m l}$ is connected to a unique $T_{n}^{*}$ and we connect all such $D_{m l}$ to $T_{n}^{*}$, thus forming $D_{m+1 n}$. We remark that any $D_{m l}$ may not be connected to some $T_{n}^{*}$ and that $T_{n}^{*}$ may be even a singleton of $y_{m+1 n}$. Then we add all open edges of $G_{m+1} \backslash \bigcup_{n} T_{n}^{*}$ to the list of $A_{i}$. We remark that the size of $A_{i}$ added in the $(m+1)$ st step is less than $1 /(m+1)$.

Continuing, we have $D_{m k}$ which are contained in a unique $D_{m+1 k^{\prime}}$. Since we pick $y_{m k}$ in each separated small area, a sequence consisting of $y_{m k} \mathrm{~s}$ converges to one point in $X^{w}$. We enumerate these points as $x_{n}$ and let the increasing union of $D_{m k}$ for which $y_{m k}$ converges to $x_{n}$, together with the singleton $\left\{x_{n}\right\}$, be $D_{n}$, which is a dendrite by construction. For each $m$, there 
are only finitely many $D_{n}$ which contain $y_{m k}$ for some $k$, and if $D_{n}$ does not contain $y_{m k}$ for any $k$, then $D_{n}$ is contained in some $\overline{P_{0}} \cup \overline{P_{1}}$ for $P_{0}, P_{1} \in \mathcal{P}_{m}$ and hence $\operatorname{diam}\left(D_{n}\right) \leq 2 / m$. For each $P \in \mathcal{P}_{m}$ with $\bar{P} \cap X^{w}=\emptyset$, we have a strong deformation retraction from $\bar{P}$ to $T_{P}$. Since $\lim _{n \rightarrow \infty} \operatorname{Mesh}\left(\mathcal{P}_{n}\right)=0$, we can take a union of strong deformation retractions of $\bar{P}$ to $T_{P}$ as a strong deformation retraction of $X$ to $X^{w} \cup \bigcup_{n} D_{n} \cup \bigcup_{i} A_{i}$. Now we have achieved the first goal.

Let $X_{0}=X^{w} \cup \bigcup_{n} D_{n} \cup \bigcup_{i} A_{i}$. Since $x_{m}=x_{n}$ may happen for $m \neq n$, we form a union of $D_{n}$ s when $x_{m}=x_{n}$. It is still a dendrite. Hence we suppose that $D_{m} \cap D_{n}=\emptyset$ for $m \neq n$. Let $Z$ be the quotient space of $X_{0}$ obtained by regarding each $D_{n}$ as one point. Since $\lim _{n \rightarrow \infty} \operatorname{diam}\left(D_{n}\right)=0$, $Z$ is a compact metrizable space (see [5, Propositions I.2.2 and I.2.3]). Since $Z$ is a countable sum of one-dimensional closed sets $X^{w}$ and $\overline{A_{i}} \mathrm{~s}, Z$ is onedimensional by [12, Theorem 7.2.1] and hence $Z$ is a one-dimensional Peano continuum. The remaining task is to show that $X_{0}$ is homotopy equivalent to $Z$.

Let $f: X_{0} \rightarrow Z$ be the quotient map. To define $g: Z \rightarrow X_{0}$, we take strong contractions $r_{n}: D_{n} \times[0,1] \rightarrow D_{n}$ such that $r_{n}\left(x_{n}, t\right)=x_{n}$ and $r_{n}(u, 1)=x_{n}$ for each $u \in D_{n}$, and we take continuous maps $a_{i}:[0,1] \rightarrow \overline{A_{i}}$ so that $a_{i}(0)=A_{i}^{0}$ and $a_{i}(1)=A_{i}^{1}$ and $a_{1} \uparrow(0,1)$ is a homeomorphism.

We define $g(u)=u$ for $u \in X^{w}$ and so it suffices to define $g(u)$ for $u$ in each $A_{i}$. There exists a unique $n_{0}$ such that $A_{i}^{0} \in D_{n_{0}}$ and also a unique $n_{1}$ such that $A_{i}^{1} \in D_{n_{1}}$. Define $g$ on $A_{i}$ by

$$
g(u)= \begin{cases}r_{n_{0}}\left(A_{i}^{0}, 1-3 s\right) & \text { if } u=a_{i}(s) \text { for } 0 \leq s \leq 1 / 3, \\ a_{i}(3 s-1) & \text { if } u=a_{i}(s) \text { for } 1 / 3<s<2 / 3 \\ r_{n_{1}}\left(A_{i}^{1}, 3 s-2\right) & \text { if } u=a_{i}(s) \text { for } 2 / 3 \leq s \leq 1\end{cases}
$$

The continuity of $g$ on $\bigcup_{i} A_{i}$ is obvious and so we consider the continuity at $x \in X^{w}$. For an open neighborhood $U$ of $g(x)=x$ in $X_{0}$, choose a neighborhood $U_{0}$ of $x$ in $X_{0}$ so that $\overline{U_{0}} \subseteq U$. Let $I_{0}=\left\{i: A_{i}^{0} \in D_{n}\right.$ or $\left.A_{i}^{1} \in D_{n}, x_{n} \in U_{0}\right\}$. There exist at most finitely many $x_{n} \in U_{0}$ for which the set $D_{n} \cup \bigcup\left\{A_{i}: A_{i}^{0} \in D_{n}\right.$ or $\left.A_{i}^{1} \in D_{n}\right\}$ is not contained in $U$. For such an $x_{n} \in U_{0}$, consider the connected component $C_{n}$ of $U \cap\left(D_{n} \cup \bigcup\left\{A_{i}: A_{i}^{0} \in D_{n}\right.\right.$ or $\left.\left.A_{i}^{1} \in D_{n}\right\}\right)$ containing $x_{n}$. Then there exist at most finitely many $A_{i}$ such that $A_{i}^{0} \in D_{n}$ or $A_{i}^{1} \in D_{n}$ and $\overline{A_{i}} \not \subseteq C_{n}$. Collecting these we have an at most finite subset $I_{1}$ of $I_{0}$ such that $g\left(A_{i}\right) \subseteq U$ for all $i \in I_{0} \backslash I_{1}$.

Since $\left(U_{0} \cap X^{w}\right) \cup \bigcup_{i \in I_{0}} A_{i}$ is an open neighborhood of $x$ in $Z$, just shrinking on $A_{i}$ for $i \in I_{1}$ we have the desired neighborhood $V_{0}$ of $x$ such that $g\left(V_{0}\right) \subseteq U$, i.e. $g$ is continuous at $x$. Since it is comparatively easy to prove $f \circ g$ is homotopic to $\mathrm{id}_{Z}$, we only prove that $g \circ f$ is homotopic to $\operatorname{id}_{X_{0}}$. 
Define $H: X_{0} \times[0,1] \rightarrow X_{0}$ by

$$
\begin{aligned}
& H(x, 0)=x \quad \text { for } x \in X_{0} \text {, } \\
& H(x, t)=x \quad \text { for } x \in X^{w} \text {, } \\
& H(x, t)=r_{n}(x, t) \quad \text { for } x \in D_{n} \text {, }
\end{aligned}
$$

and

$$
\begin{aligned}
& H\left(a_{i}(s), t\right) \\
& = \begin{cases}r_{n_{i 0}}\left(A_{i}^{0}, t-s-2 s t\right) & \text { if } 0 \leq s \leq 1 / 3 \text { and } s+2 s t-t<0, \\
a_{i}(s+2 s t-t) & \text { if } 0 \leq s \leq 1 / 3 \text { and } s+2 s t-t \geq 0, \\
a_{i}(s+2 s t-t) & \text { if } 1 / 3 \leq s \leq 2 / 3, \\
a_{i}(s+2 s t-t) & \text { if } 2 / 3 \leq s \leq 1 \text { and } s+2 s t-t \leq 1, \\
r_{n_{i 1}}\left(A_{i}^{1}, s+2 s t-t-1\right) & \text { if } 2 / 3 \leq s \leq 1 \text { and } s+2 s t-t>1 .\end{cases}
\end{aligned}
$$

Then $H(x, 1)=g \circ f(x)$ for $x \in X_{0}$. The continuity of $H$ at $(x, t)$ for $x \in X^{w}$ is shown by similar considerations to those for $g$.

REMARK 2.3. We remark a difference between the proof of 8 , Theorem 1.2] and the above one. In the former case $D_{m k} \mathrm{~s}$ and $A_{i} \mathrm{~s}$ converge to one point automatically and the care for the sizes of connecting paths between $y_{m l}$ and $y_{m+1 n}$ is not necessary. But in the above case, if we do not take care, we might not be able to find the end point of $D_{n}$ in $X^{w}$. So, we connect them in each $\bar{P}$ for $P \in \mathcal{P}_{m}$ separately.

3. Lemmas for paths. First we recall some material from [8].

Lemma 3.1 ([8, Lemma 5.1]). Let $X$ be a first countable space and $Y$ be a one-dimensional metric space and $h: \pi_{1}(X, x) \rightarrow \pi_{1}(Y, y)$ be a homomorphism. Then for $x_{0} \in X_{h}^{w}$ there exists a unique point $y_{0} \in Y$ which satisfies the following condition:

for a path $p:[0,1] \rightarrow X$ with $p(0)=x_{0}$ and $p(1)=x$, there exists a unique path $q:[0,1] \rightarrow Y$ from $y_{0}=q(0)$ to $y=q(1)$ up to homotopy which satisfies the following:

for each continuous map $f:(\mathbb{H}, o) \rightarrow\left(X, x_{0}\right)$ there exists a continuous map $g:(\mathbb{H}, o) \rightarrow\left(Y, y_{0}\right)$ such that $h \circ \varphi_{p} \circ f_{*}=\varphi_{q} \circ g_{*}$.

Using this lemma we defined $\tilde{h}: X_{h}^{w} \rightarrow Y$ in [8, p. 497]. We want to extend $\tilde{h}$ to $X$. For this purpose we recall the setting from [8, Section 6]. Here we generalize it a little.

For a one-dimensional space $X$, a point $x \in X$ and a subset $S$ of $X$,

- $P(X)$ is the set of all paths in $X$,

- $P_{x}(X)$ is the set of all paths which terminate at $x$,

- $P_{S}(X)$ is the set of all paths which connect points in $S$, 
- $P_{S, x}(X)$ is the set of all paths which start from points in $S$ and terminate at $x$,

- $P^{h}(X)$ is the set of all homotopy classes of paths in $X$,

- $R P(X)$ is the set of all reduced paths in $X$,

- $R P_{x}(X)$ is the set of all reduced paths in $X$ which terminate at $x$,

- $P_{x}^{h}(X)$ is the homotopy classes that are represented by the paths in $P_{x}(X)$.

Since any path is homotopic to a reduced path (see [4]), there is a one-to-one correspondence between $P^{h}(X)$ (or $\left.P_{x}^{h}(X)\right)$ and the quotient of $R P(X)$ (or $\left.R P_{x}(X)\right)$ modulo equivalence. According to our definition of homotopies between paths, homotopies are relative to end points, the initial point and the terminal point of the class $[p]$ are well-defined for a homotopy class $[p] \in P^{h}(X)$. If $p q$ is a path for two paths $p$ and $q$, then $[p][q]$ is defined as $[p q]$. An element of $P^{h}(X)$ is degenerate if it is the equivalence class of a degenerate path.

For an open set $U$ containing the initial point of $[p]$, let $O(U,[p])=\{[f]$ : $f$ is homotopic to $g p$ for some $g$ with $\operatorname{Im}(g) \subseteq U\}$. The tail-limit topology is the topology on $P_{x}^{h}(X)$ which has the collection of all $O(U,[p])$ 's as a neighborhood base for $[p]$. Let $\sigma: P_{x}^{h}(X) \rightarrow X$ be the map which sends $[p]$ to the initial point of $p$.

Lemma 3.2 ([즈, Lemma 6.6]). Let $X$ be a one-dimensional metric space and $F:[0,1] \rightarrow P_{x}^{h}(X)$ be a path such that $F(0)$ is degenerate. If $f \in$ $R P_{x}(X)$ represents $F(1)$, then $\sigma \circ F$ and $f^{-}$are homotopic.

We remark that this statement was wrongly stated as " $\sigma \circ F$ and $f$ are homotopic" in [8].

For a homomorphism $h: \pi_{1}(X, x) \rightarrow \pi_{1}(Y, y)$, we define $\psi: P_{X_{h}^{w}, x}(X) \rightarrow$ $R P_{y}(Y)$ and $\xi: P_{X_{h}^{w}}(X) \rightarrow R P(Y)$ as follows. For a path $p$ from $x_{0} \in X_{h}^{w}$ to $x$, we have a reduced path $q$ from $\tilde{h}\left(x_{0}\right)$ to $y$ according to Lemma 3.1 such that the properties there hold. We define $\psi(p)=q$. For a path $p_{0}$ from $x_{1} \in X_{h}^{w}$ to $x_{0} \in X_{h}^{w}$ in $X, p_{0} p$ is a path from $x_{1}$ to $x$. We define $\xi\left(p_{0}\right)$ to be a reduced path homotopic to $\psi\left(p_{0} p\right) \psi(p)^{-}$. We remark that in case $x=x_{0}, \psi\left(p_{0}\right)$ and $\xi\left(p_{0}\right)$ are defined, but may be distinct. In particular, for the constant loop $c_{x}$ at $x, \xi\left(c_{x}\right)$ is a degenerate path, but $\psi\left(c_{x}\right)$ may not be.

Lemma 3.3. Let $p$ and $p^{\prime}$ be paths from $x_{0} \in X_{h}^{w}$ to $x$. Then $h\left(\left[p^{-} p^{\prime}\right]\right)=$ $\left[\psi(p)^{-} \psi\left(p^{\prime}\right)\right]$. Consequently, for a loop $l$ with base point $x_{0}, h \circ \varphi_{p}([l])=$ $\varphi_{\psi(p)}([\xi(l)])$.

Proof. Let $q=\psi(p)$ and $q^{\prime}=\psi\left(p^{\prime}\right)$. Then, as in Lemma 3.1, for each continuous $f:(\mathbb{H}, o) \rightarrow\left(X, x_{0}\right)$ there is a continuous map $g:(\mathbb{H}, o) \rightarrow\left(Y, y_{0}\right)$ such that $h \circ \varphi_{p p^{-} p^{\prime}} \circ f_{*}=h \circ \varphi_{p^{\prime}} \circ f_{*}=\varphi_{q^{\prime}} \circ g_{*}$. Since $h \circ \varphi_{p p^{-} p^{\prime}}\left(f_{*}([u])\right)=$ $h\left(\left[\left(p^{-} p^{\prime}\right)^{-}\right]\right) h\left(\left[p^{-}(f \circ u) p\right]\right) h\left(\left[p^{-} p^{\prime}\right]\right)$ for a loop $u:[0,1] \rightarrow \mathbb{H}$ with $u(0)=$ 
$u(1)=o$, we have

$$
\begin{aligned}
h\left(\left[p^{-} p^{\prime}\right]\right)\left[q^{\prime-}\right][g \circ u]\left[q^{\prime}\right] h\left(\left[p^{-} p^{\prime}\right]\right)^{-1} & =h\left(\left[p^{-} p^{\prime}\right]\right) \varphi_{q^{\prime}}\left(g_{*}([u])\right) h\left(\left[p^{-} p^{\prime}\right]\right)^{-1} \\
& =h\left(\left[p^{-} p^{\prime}\right]\right) h \circ \varphi_{p p^{-} p^{\prime}} \circ f_{*}([u]) h\left(\left[p^{-} p^{\prime}\right]^{-1}\right) \\
& =h\left(\left[p^{-}(f \circ u) p\right]\right) \\
& =\varphi_{q}\left(g_{*}([u])\right)=\left[q^{-}\right][g \circ u][q] .
\end{aligned}
$$

Since $q$ is unique up to homotopy, we have $h\left(\left[p^{-} p^{\prime}\right]\right)\left[q^{-}\right]=\left[q^{-}\right]$and hence $h\left(\left[p^{-} p^{\prime}\right]\right)=\left[q^{-} q^{\prime}\right]=\left[\psi(p)^{-} \psi\left(p^{\prime}\right)\right]$.

Next let $p^{\prime}=l p$. Then we have

$$
\begin{aligned}
h \circ \varphi_{p}([l]) & =h\left(\left[p^{-} p^{\prime}\right]\right)=\left[\psi(p)^{-} \psi(l p)\right]=\left[\psi(p)^{-} \psi(l p) \psi(p)^{-} \psi(p)\right] \\
& =\varphi_{\psi(p)}([\xi(l)]) .
\end{aligned}
$$

Lemma 3.4. The definition of $\xi\left(p_{0}\right)$ does not depend on $p$. More precisely $\xi\left(p_{0}\right)$ is defined by the homotopy class $\left[p_{0}\right]$ of $p_{0}$ and $h$ uniquely up to the equivalence.

Proof. To see this let $p^{\prime}$ be another path from $x_{0}$ to $x$. By Lemma 3.3 , $h\left(\left[p^{-} p^{\prime}\right]\right)=\left[\psi(p)^{-} \psi\left(p^{\prime}\right)\right]$ and $h\left(\left[\left(p_{0} p^{\prime}\right)^{-}\left(p_{0} p\right)\right]\right)=\left[\psi\left(p_{0} p^{\prime}\right)^{-} \psi\left(p_{0} p\right)\right]$. Thus

$$
\left[\psi\left(p_{0} p^{\prime}\right)^{-} \psi\left(p_{0} p\right)\right]=h\left(\left[\left(p_{0} p^{\prime}\right)^{-}\left(p_{0} p\right)\right]\right)=h\left(\left[p^{\prime-} p\right]\right)=\left[\psi\left(p^{\prime}\right)^{-} \psi(p)\right]
$$

and hence $\left[\psi\left(p_{0} p^{\prime}\right)^{-} \psi\left(p_{0} p\right) \psi(p)^{-} \psi\left(p^{\prime}\right)\right]=e$, which implies that

$$
\xi\left(p_{0}\right) \sim \psi\left(p_{0} p\right) \psi(p)^{-} \sim \psi\left(p_{0} p^{\prime}\right) \psi\left(p^{\prime}\right)^{-} .
$$

LEMMA 3.5. Let $x_{0}, x_{1}, x_{2} \in X_{h}^{w}$ and $p_{0}$ be a path from $x_{1}$ to $x_{0}$ and $p_{1}$ be a path from $x_{2}$ to $x_{1}$ and $p$ be a path from $x_{0}$ to $x$. Then $\psi\left(p_{0} p\right) \sim \xi\left(p_{0}\right) \psi(p)$ and $\xi\left(p_{1} p_{0}\right) \sim \xi\left(p_{1}\right) \xi\left(p_{0}\right)$.

Proof. Since $\xi\left(p_{0}\right) \sim \psi\left(p_{0} p\right) \psi(p)^{-}$, we have $\psi\left(p_{0} p\right) \sim \xi\left(p_{0}\right) \psi(p)$. Now $\xi\left(p_{1} p_{0}\right) \sim \psi\left(p_{1} p_{0} p\right) \psi(p)^{-}$and $\xi\left(p_{1}\right) \sim \psi\left(p_{1} p_{0} p\right) \psi\left(p_{0} p\right)^{-}$by Lemma 3.4. Hence

$$
\xi\left(p_{1} p_{0}\right) \sim \xi\left(p_{1}\right) \psi\left(p_{0} p\right) \psi(p)^{-} \sim \xi\left(p_{1}\right) \xi\left(p_{0}\right) .
$$

LEMMA 3.6. Let $X, Y$ and $Z$ be one-dimensional metric spaces and $g$ : $\pi_{1}(X, x) \rightarrow \pi_{1}(Y, y)$ and $h: \pi_{1}(Y, y) \rightarrow \pi_{1}(Z, z)$ be homomorphisms. Let $\psi_{0}: P_{X_{g}^{w}, x}(X) \rightarrow R P_{y}(Y), \xi_{0}: P_{X_{g}^{w}}(X) \rightarrow R P(Y), \psi_{1}: P_{Y_{h}^{w}, y}(Y) \rightarrow$ $R P_{z}(Z), \xi_{1}: P_{Y_{h}^{w}}(Y) \rightarrow R P(Z)$ and $\psi_{2}: P_{X_{h \circ g}^{w}, x}(X) \rightarrow R P_{z}(Z), \xi_{2}:$ $P_{X_{h \circ g}^{w}}(X) \rightarrow R P(Z)$ be the maps induced from $g$, $h$ and $h \circ g$ respectively. Then $\psi_{1}\left(\psi_{0}(p)\right) \sim \psi_{2}(p)$ for $p \in P_{X_{g}^{w}, x}(X)$, and $\xi_{1}\left(\xi_{0}\left(p_{0}\right)\right) \sim \xi_{2}\left(p_{0}\right)$ for $p_{0} \in P_{X_{h \circ g}^{w}}(X)$.

Proof. We remark that $X_{h \circ g}^{w} \subseteq X_{g}^{w}$ and $\tilde{g}\left(x_{0}\right) \in Y_{h}^{w}$ for $x_{0} \in X_{h \circ g}^{w}$.

Since $\psi_{0}(p)$ is determined by a continuous map $f:(\mathbb{H}, o) \rightarrow\left(X, x_{0}\right)$ such that $\operatorname{Im}\left(f_{*}\right)$ is infinitely generated instead of a continuous map from $(\mathbb{H}, o)$ to $\left(Y, y_{0}\right)$, we easily get $\psi_{1}\left(\psi_{0}(p)\right) \sim \psi_{2}(p)$ for $p \in P_{X_{g}^{w}, x}$. 
Now we have $\psi_{1}\left(\psi_{0}\left(p_{0} p\right)\right) \sim \psi_{2}\left(p_{0} p\right)$ and hence

$$
\xi_{1}\left(\xi_{0}\left(p_{0}\right)\right) \psi_{1}\left(\psi_{0}(p)\right) \sim \psi_{1}\left(\xi_{0}\left(p_{0}\right) \psi_{0}(p)\right) \sim \psi_{1}\left(\psi_{0}\left(p_{0} p\right)\right) \sim \xi_{2}\left(p_{0}\right) \psi_{2}(p)
$$

by Lemma 3.5. It follows that $\xi_{1}\left(\xi_{0}\left(p_{0}\right)\right) \sim \xi_{2}\left(p_{0}\right)$.

The next lemma strengthens the continuity of $\tilde{h}$ on $X_{h}^{w}$ [8, Lemma 5.3] and hence its proof is a modification of that of [8, Lemma 5.3].

Lemma 3.7. Let $X$ and $Y$ be one-dimensional metric spaces, $X$ be locally path-connected and $h: \pi_{1}(X, x) \rightarrow \pi_{1}(Y, y)$ be a homomorphism. Let $x_{n} \in$ $X_{h}^{w}$ and $p_{n}$ be a path from $x_{n}$ to $x_{\infty}$ for each $n<\omega$ such that $\operatorname{Im}\left(p_{n}\right)$ converges to $x_{\infty} \in X_{h}^{w}$. Then $\operatorname{Im}\left(\xi\left(p_{n}\right)\right)$ converges to $\tilde{h}\left(x_{\infty}\right)$.

Proof. Aiming for a contradiction, suppose that there is a neighborhood $U$ of $\tilde{h}\left(x_{\infty}\right)$ such that $\operatorname{Im}\left(\xi\left(p_{n}\right)\right) \nsubseteq U$ for all $n$. Let $p$ be a path from $x_{\infty}$ to $x$. According to [8, Lemma 6.1] we have an arbitrary small loop $l_{n}$ with base point $x_{n}$ such that $h \circ \varphi_{p_{n} p}\left(\left[l_{n}\right]\right)$ is represented as a reduced loop $\psi\left(p_{n} p\right)^{-} l_{n}^{\prime} \psi\left(p_{n} p\right)$ for a cyclically reduced loop $l_{n}^{\prime}$. Hence we have a continuous map $f:(\mathbb{H}, o) \rightarrow\left(X, x_{\infty}\right)$ such that $f \circ \mathbf{e}_{n}=p_{n}^{-} l_{n} p_{n}$. Lemma 3.1.implies that we have a path $q$ from $\tilde{h}\left(x_{\infty}\right)$ to $y$ such that $h \circ \varphi_{p} \circ f_{*}=\varphi_{q} \circ g_{*}$ for a continuous map $g:(\mathbb{H}, o) \rightarrow\left(Y, \tilde{h}\left(x_{\infty}\right)\right)$. For a sufficiently large $n$, we have $\operatorname{Im}\left(g \circ \mathbf{e}_{n}\right) \subseteq U$. On the other hand,

$$
\begin{aligned}
\varphi_{q} \circ g_{*}\left(\left[\mathbf{e}_{n}\right]\right) & =h \circ \varphi_{p} \circ f_{*}\left(\left[\mathbf{e}_{n}\right]\right)=h \circ \varphi_{p}\left(\left[p_{n}^{-} l_{n} p_{n}\right]\right)=h \circ \varphi_{p_{n} p}\left(\left[l_{n}\right]\right) \\
& =\left[\psi\left(p_{n} p\right)^{-} l_{n}^{\prime} \psi\left(p_{n} p\right)\right]=\left[\psi(p)^{-} \xi\left(p_{n}\right)^{-} l_{n}^{\prime} \xi\left(p_{n}\right) \psi(p)\right] \\
& =\varphi_{q}\left(\left[\xi\left(p_{n}\right)^{-} l_{n}^{\prime} \xi\left(p_{n}\right)\right]\right)
\end{aligned}
$$

and hence $\left[g \circ \mathbf{e}_{n}\right]=\left[\xi\left(p_{n}\right)^{-} l_{n}^{\prime} \xi\left(p_{n}\right)\right]$. Since $\xi\left(p_{n}\right)$ is a reduced path and $l^{\prime}$ is cyclically reduced, one of $\xi\left(p_{n}\right)^{-} l^{\prime}$ and $l^{\prime} \xi\left(p_{n}\right)$ is reduced. Hence the image of the reduced loop of $\xi\left(p_{n}\right)^{-} l_{n}^{\prime} \xi\left(p_{n}\right)$ is not contained in $U$ by [8, Lemma 2.6]. Now $\xi\left(p_{n}\right)^{-} l_{n}^{\prime} \xi\left(p_{n}\right)$ is not homotopic to a loop in $U$ (see the first three lines of Section 4 ) and we have a contradiction.

Lemma 3.8. Let $X$ and $Y$ be one-dimensional metric spaces and $X$ be locally path-connected and path-connected, and $h: \pi_{1}(X, x) \rightarrow \pi_{1}(Y, y)$ be a homomorphism. Let $x_{n}, y_{n} \in X_{h}^{w}$ and $p_{n}$ be a path from $y_{n}$ to $x_{n}$ for each $n<\omega$ such that $\operatorname{Im}\left(p_{n}\right)$ converges to $x_{\infty} \in X_{h}^{w}$. Then $\operatorname{Im}\left(\xi\left(p_{n}\right)\right)$ converges to $\tilde{h}\left(x_{\infty}\right)$.

Proof. By the path-connectivity and local path-connectivity, we have a path $f_{n}$ from $x_{n}$ to $x_{\infty}$ for each $n$ such that $\operatorname{Im}\left(f_{n}\right)$ converges to $x_{\infty}$. As in the proof of Lemma 3.7, suppose there is a neighborhood $U$ of $\tilde{h}\left(x_{\infty}\right)$ such that $\operatorname{Im}\left(\xi\left(p_{n}\right)\right) \nsubseteq U$ for all $n$. Since $\xi\left(p_{n}\right) \sim \xi\left(p_{n} f_{n} f_{n}^{-}\right) \sim \xi\left(p_{n} f_{n}\right) \xi\left(f_{n}\right)^{-}$, we have $\operatorname{Im}\left(\xi\left(f_{n}\right)\right) \nsubseteq U$ or $\operatorname{Im}\left(\xi\left(p_{n} f_{n}\right)\right) \nsubseteq U$. We choose $x_{n}$ if $\operatorname{Im}\left(f_{n}\right) \nsubseteq U$, and $y_{n}$ otherwise. Then we have paths contradicting Lemma 3.7 . 
Let $X$ be a locally path-connected, path-connected, one-dimensional metric space, $Y$ a one-dimensional metric space and suppose that for a homomorphism $h: \pi_{1}(X, x) \rightarrow \pi_{1}(Y, y), X=X_{h}^{w} \cup \bigcup_{i \in I} A_{i}$, where $A_{i}$ s are open arcs and $A_{i}^{0}, A_{i}^{1} \in X_{h}^{w}$. Note that $\lim _{i \rightarrow \infty} \operatorname{diam}\left(A_{i}\right)=0$. Then $\tilde{h}: X_{h}^{w} \rightarrow Y$ is a continuous map by [8, Lemma 5.3]. We extend $\tilde{h}$ on $X$ as follows. For each $A_{i}$, we choose a continuous map $a_{i}:[0,1] \rightarrow \overline{A_{i}}$ with $a_{i}(0)=A_{i}^{0}$ and $a_{i}(1)=A_{i}^{1}$ so that the restriction of $a_{i}$ to $(0,1)$ is injective. (That is, $a_{i}$ is a homeomorphism if $A_{i}^{0} \neq A_{i}^{1}$.) Then define $\tilde{h}(x)=\xi\left(a_{i}\right)\left(a_{i}^{-1}(x)\right)$ for $x \in A_{i}$.

Then the continuity of $\tilde{h}$ on $\bigcup_{i \in I} A_{i}$ is obvious and that at each point in $X_{h}^{w}$ follows from Lemma 3.8, since $\lim _{n \rightarrow \infty} \operatorname{diam}\left(A_{n}\right)=0$. We use this extended $\tilde{h}$ in Lemmas 3.9 and 3.10 and also in the proofs of Theorems 1.1 and 1.2 . In Lemma 3.10 we suppose that $Y$ also has the additional properties which $X$ has.

LEMma 3.9. Let $h: \pi_{1}(X, x) \rightarrow \pi_{1}(Y, y)$ be a homomorphism and $r$ be a reduced path from $x_{1} \in X_{h}^{w}$ to $x_{0} \in X_{h}^{w}$. Then $\xi(r)$ is homotopic to $\tilde{h} \circ r$.

Proof. Let $p_{t}$ be the restriction of $r$ to $[1-t, 1]$ for $0 \leq t \leq 1$, e.g. $p_{1}=r$ and $p_{0}$ is the degenerate path at $x_{0}$. Define $F:[0,1] \rightarrow P_{\tilde{h}\left(x_{0}\right)}^{h}(Y)$ as follows. Let $F(t)=\left[\xi\left(p_{t}\right)\right]$ if $\sigma\left(\left[p_{t}\right]\right) \in X_{h}^{w}$. Otherwise, we have $i \in I$ and $0 \leq t_{0}<t_{1} \leq 1$ such that $t_{0}<t<t_{1}, \sigma\left(\left[p_{t_{0}}\right]\right), \sigma\left(\left[p_{t_{1}}\right]\right) \in X^{w}$ and $r \uparrow\left[1-t_{1}, 1-t_{0}\right] \sim a_{i}$ or $r \uparrow\left[1-t_{1}, 1-t_{0}\right] \sim a_{i}^{-}$. Let

$$
F(t)= \begin{cases}{\left[\xi\left(a_{i} \uparrow\left[\left(t_{1}-t\right) /\left(t_{1}-t_{0}\right), 1\right]\right) \xi\left(p_{t_{0}}\right)\right]} & \text { if } r \uparrow\left[1-t_{1}, 1-t_{0}\right] \sim a_{i}, \\ {\left[\xi\left(a_{i}^{-} \uparrow\left[\left(t_{1}-t\right) /\left(t_{1}-t_{0}\right), 1\right]\right) \xi\left(p_{t_{0}}\right)\right]} & \text { if } r \uparrow\left[1-t_{1}, 1-t_{0}\right] \sim a_{i}^{-} .\end{cases}
$$

If $\sigma\left(\left[p_{t}\right]\right) \notin X_{h}^{w}$, the continuity of $F$ at $t$ is obvious. Otherwise, the continuity of $F$ at $t$ follows from Lemma 3.8 . Since $F(1)=[\xi(r)]$ and $\tilde{h}(r(1-t))=$ $\sigma \circ F(t)$, the conclusion follows from Lemma 3.2 .

LEMmA 3.10. Let $h_{0}: \pi_{1}(X, x) \rightarrow \pi_{1}(Y, y)$ be an isomorphism and $h_{1}$ be its inverse. Let $p$ be a path between points in $X^{w}$. Then $p$ is homotopic to $\tilde{h}_{1} \circ \tilde{h}_{0} \circ p$. In particular $\tilde{h}_{1} \circ \tilde{h}_{0}\left(x_{0}\right)=x_{0}$ for $x_{0} \in X^{w}$.

Proof. Since every path is homotopic to a reduced path, it suffices to show this lemma for a reduced path $p$. Let $\xi_{0}: P_{X w}(X) \rightarrow R P(Y)$ and $\xi_{1}: P_{Y^{w}}(Y) \rightarrow R P(X)$ be the maps induced from $h_{0}$ and $h_{1}$ respectively. We remark that $X_{h_{0}}^{w}=X^{w}$ and $Y_{h_{1}}^{w}=Y^{w}$. Then $\left[\xi_{1}\left(\xi_{0}(p)\right)\right]=[p]$ by Lemma 3.6. The conclusion follows from Lemma 3.9.

4. Proofs of Theorems 1.1 and 1.2 . The following lemma is wellknown and can be proved if we notice that any path is homotopic to a reduced path in its image [4] and the reduced path is unique up to equivalence. 
Lemma 4.1 (Folklore). Let $X$ be a one-dimensional space. Then two homotopic paths in $X$ are homotopic in the union of their ranges.

Proof of Theorem 1.1. By Theorem 2.1 we may assume that $X=X^{w} \cup$ $\bigcup_{i \in I} A_{i}$ and $Y=Y^{w} \cup \bigcup_{j \in J} B_{j}$, where $I$ and $J$ are at most countable, $A_{i}$ and $B_{j}$ are open arcs. Let $h_{0}: \pi_{1}(X, x) \rightarrow \pi_{1}(Y, y)$ be an isomorphism and $h_{1}: \pi_{1}(Y, y) \rightarrow \pi_{1}(X, x)$ be its inverse. We assume that $\tilde{h}_{0}$ and $\tilde{h}_{1}$ are extensions on $X$ and $Y$ respectively according to the description before Lemma 3.9. By Lemma 3.10, $a_{i}$ and $\tilde{h}_{1} \circ \tilde{h}_{0} \circ a_{i}$ are homotopic for each $i$. By Lemma 4.1 we have a homotopy $H_{i}:[0,1] \times[0,1] \rightarrow X$ such that

$$
\begin{aligned}
H_{i}(s, 0) & =a_{i}(s), & H_{i}(s, 1) & =\tilde{h}_{1} \circ \tilde{h}_{0} \circ a_{i}(s), \\
H_{i}(0, t) & =a_{i}(0), & H_{i}(1, t) & =a_{i}(1)
\end{aligned}
$$

and $\operatorname{Im}\left(H_{i}\right) \subset \operatorname{Im}\left(a_{i}\right) \cup \operatorname{Im}\left(\tilde{h}_{1} \circ \tilde{h}_{0} \circ a_{i}\right)$. Since $\lim _{i \rightarrow \infty} \operatorname{diam}\left(A_{i}\right)=0$, we have $\lim _{i \rightarrow \infty} \operatorname{diam}\left(\operatorname{Im}\left(H_{i}\right)\right)=0$. Define $\bar{H}_{i}: A_{i} \times[0,1] \rightarrow X$ by $\bar{H}_{i}(x, t)=$ $H_{i}\left(a_{i}^{-1}(x), t\right)$. Then

$$
\begin{array}{ll}
\bar{H}_{i}(x, 0)=a_{i}\left(a_{i}^{-1}(x)\right)=x, & \bar{H}_{i}\left(A_{i}^{0}, t\right)=a_{i}(0)=A_{i}^{0}, \\
\bar{H}_{i}\left(A_{i}^{1}, t\right)=a_{i}(1)=A_{i}^{1}, & \bar{H}_{i}(x, 1)=\tilde{h}_{1} \circ \tilde{h}_{0} \circ a_{i}\left(a_{i}^{-1}(x)\right)=\tilde{h}_{1} \circ \tilde{h}_{0}(x) .
\end{array}
$$

Define $\bar{H}: X \times[0,1] \rightarrow X$ by $\bar{H}(x, t)=x$ for $x \in X^{w}$ and $0 \leq t \leq 1$ and $\bar{H}(x, t)=\bar{H}_{i}(x, t)$ for $x \in A_{i}$ and $0 \leq t \leq 1$. Then the continuity of $\bar{H}$ follows from that of all $\bar{H}_{i}$ and the fact that $\lim _{i \rightarrow \infty} \operatorname{diam}\left(\operatorname{Im}\left(\bar{H}_{i}\right)\right)=0$. Hence $\tilde{h}_{1} \circ \tilde{h}_{0}$ is homotopic to the identity map on $X$ and similarly $\tilde{h}_{0} \circ \tilde{h}_{1}$ is homotopic to the identity map on $Y$.

For our proof of Theorem 1.2 one more notion is necessary. Let $h$ : $\pi_{1}(x, x) \rightarrow G$ be a homomorphism. We call a subset $S$ of $X$ h-simply connected if for every point $x_{0}$ in $S$, every loop $l$ in $S$ with base point $x_{0}$ and every path $q$ from $x_{0}$ to $x, h\left(\varphi_{q}([l])\right)$ is trivial. We remark that if $h\left(\varphi_{q}([l])\right)$ is trivial, then $h\left(\varphi_{q^{\prime}}([l])\right)$ is also trivial for every path $q^{\prime}$ from $x_{0}$ to $x$.

Proof of Theorem 1.2. If $X_{h}^{w}$ is empty, then $O_{h}^{X}=X$ and we have a brick partition $\mathcal{P}$ such that

- $\mathcal{P}$ is of order 2 ;

- $\bar{P}$ is $h$-simply connected for $P \in \mathcal{P}$;

- if $\bar{P} \cap \bar{Q} \neq \emptyset$, then $\bar{P} \cup \bar{Q}$ is $h$-simply connected for $P, Q \in \mathcal{P}$.

Hence this is the case when our procedure stops at the first step and we can easily get the conclusion from the following arguments. Hence we proceed to the case when $X_{h}^{w} \neq \emptyset$. We construct a retract $K \cup X_{h}^{w}$ of $X$, where $K$ is locally homeomorphic to finite graphs in $\overline{O_{h}^{X}}$. This is a modification of the proof of Theorem 2.1 in Section 2,

Starting from the 0 th step we let $\mathcal{P}_{0}=\{X\}$, but do not define $A_{i} \mathrm{~s}$ and so on as before. We trace the previous proof, but we set buffers around 
$\bigcup\left\{\bar{P}: P \in \mathcal{P}_{m}, \bar{P} \cap X_{h}^{w} \neq \emptyset\right\}\left(=R_{m}\right)$. We call $P \in \mathcal{P}_{m}$ a buffer element if $\bar{P} \cap R_{m} \neq \emptyset$ but $P \cap R_{m}=\emptyset$.

After the $m$ th step, we have at most finitely many points $y_{m n}$ on the boundary of $\bigcup\left\{\bar{P}: P \in \mathcal{P}_{m}, \bar{P} \cap R_{m} \neq \emptyset\right\}$. Applying Proposition 2.2, we take a brick partition $\mathcal{P}_{m+1}$ of $R_{m}$ which satisfies the following:

- $\mathcal{P}_{m+1}$ is of order 2 and $\operatorname{Mesh}\left(\mathcal{P}_{m+1}\right)<1 /(m+1)$;

- $\mathcal{P}_{m+1}$ refines the restriction of $\mathcal{P}_{m}$ to $R_{m}$;

- if $P \in \mathcal{P}_{m}$ is a buffer element, then $\bar{P} \cap R_{m}$ is a disjoint union of sets of the form $\bar{P} \cap \bar{Q}$ with $Q \in \mathcal{P}_{m+1}$ and $Q \cap R_{m+1}=\emptyset$;

- if $\bar{Q} \cap \overline{Q^{\prime}} \neq \emptyset, \bar{Q} \cap X_{h}^{w}=\emptyset$ and $\overline{Q^{\prime}} \cap X_{h}^{w}=\emptyset$ for $Q, Q^{\prime} \in \mathcal{P}_{m+1}$, then $\bar{Q} \cup \overline{Q^{\prime}}$ is $h$-simply connected;

- if $\bar{Q} \cap \bar{P} \neq \emptyset$ and $\bar{Q} \cap X_{h}^{w}=\emptyset$ for $Q \in \mathcal{P}_{m+1}$ and a buffer element $P \in \mathcal{P}_{m}$, then $\bar{Q} \cup \bar{P}$ is $h$-simply connected.

We successively construct a finite tree $T_{P}$ for each buffer element $P \in \mathcal{P}_{m}$ such that end points of $T_{P}$ are chosen from $\partial P$ as follows. First we choose one point from each nonempty $\bar{P} \cap \overline{P^{\prime}}$ for $P^{\prime} \in \mathcal{P}_{m}$ with $P^{\prime} \notin R_{m}$ and $P^{\prime} \neq P$, making sure this point is among the points $y_{m n}$ whenever possible and making sure that our choice is consistent with whichever point from $\partial P^{\prime}$ might have already been chosen. Then we choose one point from each nonempty $\bar{P} \cap \bar{Q}$ with $Q \in \mathcal{P}_{m+1}$. Since $P$ is connected, we have a tree $T_{P} \subseteq \bar{P}$ such that $T_{P} \cap P$ is connected and the end points of $T_{P}$ are the elements chosen from $\partial P$. Now each end point of $T_{P}$ corresponds to some $\bar{P} \cap \overline{P^{\prime}}$ or to some $\bar{P} \cap \bar{Q}$. Since $T_{P}$ is an absolute extensor, we have a retraction $r_{P}: \bar{P} \rightarrow T_{P}$ so that $r_{P}\left(\bar{P} \cap \overline{P^{\prime}}\right)=\{v\}$ or $r_{P}(\bar{P} \cap \bar{Q})=\{v\}$ for each end point $v \in T_{P}$ with $v \in \bar{P} \cap \overline{P^{\prime}}$ or $v \in \bar{P} \cap \bar{Q}$ respectively. Next we define trees $T_{Q}$ and retractions $r_{Q}: \bar{Q} \rightarrow T_{Q}$ for $Q \in \mathcal{P}_{m+1}$ with $\bar{Q} \cap R_{m+1}=\emptyset$ just as for $P \in \mathcal{P}_{m}$. We define neither $T_{Q}$ nor $r_{Q}$ for buffer elements $Q \in \mathcal{P}_{m+1}$ in this step. But, on a part of $\partial Q$ for a buffer element $Q$ retractions $r_{P}$ or $r_{Q^{\prime}}$ have been defined. We enumerate the retracted points of those parts of boundaries as $y_{m+1 n}$ $\left(n \in I_{m+1}\right)$. More exactly, $y_{m+1 n}\left(n \in I_{m+1}\right)$ is the one-to-one enumeration of the points in $T_{P} \cap \bar{Q}$ for buffer elements $P \in \mathcal{P}_{m}$ and buffer elements $Q \in \mathcal{P}_{m+1}$ or in $T_{Q} \cap \overline{Q^{\prime}}$ for nonbuffer elements $Q \in \mathcal{P}_{m+1}$ and buffer elements $Q^{\prime} \in \mathcal{P}_{m+1}$.

For each $y_{m l} \in \bar{P}$ where $P \in \mathcal{P}_{m}$ is a buffer element, we have some $P^{\prime} \in \mathcal{P}_{m}$ such that $\bar{P} \cap \overline{P^{\prime}} \neq \emptyset$ and $\overline{P^{\prime}} \cap X^{w} \neq \emptyset$. Then we have some $y_{m+1 n}$ in $\overline{P^{\prime}}$, since $\overline{P^{\prime}} \cap X^{w} \neq \emptyset$. We can connect $y_{m l}$ and $y_{m+1 n}$ by an $\operatorname{arc}$ in $T_{P}$ and $T_{Q}$ s for $Q \subseteq P^{\prime}$. Using these arcs we construct $D_{m n}$ and $A_{i}$ similarly to the proof of Theorem 2.1 and have $D_{n}, A_{i}$ and the desired $K=\bigcup_{n} D_{n} \cup \bigcup_{i} A_{i}$. Let $X_{0}=X_{h}^{w} \cup \cup_{n} D_{n} \cup \bigcup_{i} A_{i}$ and $r: X \rightarrow X_{0}$ be the retraction obtained as the union of $r_{P}$ and the identity on $X_{h}^{w}$. 
First we assume $x \in X_{h}^{w}$. We remark $r(x)=x$. To trace our previous proof, we show $h([l])=h([r \circ l])$ for any loop $l$ with base point $x$. By [10, Theorem 1], $\pi_{1}(Y, y)$ is a subgroup of an inverse limit of free groups. It suffices to show that $g \circ h\left(\left[l^{-}(r \circ l)\right]\right)=e$ for every homomorphism $g$ from $\pi_{1}(Y, y)$ to a free group. By [9, Theorem 1.3] (cf. [3]), we have $\varepsilon>0$ such that any open connected subset of $X$ of diameter less than $\varepsilon$ is $g \circ h$ simply connected. Since $\lim _{m \rightarrow \infty} \operatorname{Mesh}\left(\mathcal{P}_{m}\right)=0$, we may choose $\mathcal{P}_{m}$ so that $\operatorname{Mesh}\left(\mathcal{P}_{m}\right)<\varepsilon / 2$. We have a brick partition $\mathcal{P}$ of $X$ such that $\mathcal{P}$ consists of $P \in \mathcal{P}_{m}$ with $\bar{P} \cap R_{m} \neq \emptyset$ and $P \in \mathcal{P}_{i}$ with $P \cap R_{i}=\emptyset$.

For a given loop $l$ with base point $x$, we have $0=u_{0}<u_{1}<\cdots<u_{k}=1$ such that $l\left(\left[u_{i}, u_{i+1}\right]\right) \subseteq \bar{P} \cup \overline{P^{\prime}}$ for $P, P^{\prime} \in \mathcal{P}$. If $\bar{P} \cap \overline{P^{\prime}} \neq \emptyset$ for $P \in \mathcal{P} \cap \mathcal{P}_{i}$ with $i<m$ and $P^{\prime} \in \mathcal{P}$, then $\bar{P} \cup \overline{P^{\prime}}$ is $h$-simply connected according to the effect of buffers. On the other hand, if $\bar{P} \cap \overline{P^{\prime}} \neq \emptyset$ for $P, P^{\prime} \in \mathcal{P} \cap \mathcal{P}_{m}$, then $\operatorname{diam}\left(\bar{P} \cup \overline{P^{\prime}}\right)<\varepsilon$ and hence $\bar{P} \cup \overline{P^{\prime}}$ is $g \circ h$-simply connected. Since each $P \in \mathcal{P}$ is path-connected, working from $k$ to 0 we see that $l^{-}(r \circ l)$ is homotopic to a concatenation of loops $l_{k} \cdots l_{1}$ such that $g \circ h\left(\left[l_{i}\right]\right)=e$. Now we have proved that $g \circ h\left(\left[l^{-}(r \circ l)\right]\right)=e$ and hence $h([r \circ l])=h([l])$. Let $i: X_{0} \hookrightarrow X$ be the inclusion map. Then for each loop $l$ in $X_{0}$ with base point $x$ we have $h \circ i_{*}([l])=h([l])$ and $X_{h}^{w} \subseteq\left(X_{0}\right)_{h \circ i_{*}}^{w} \subseteq X_{0}^{w} \subseteq X_{h}^{w}$.

The space $Z$ obtained from this $X_{0}$ as in the proof in Section 2 is a one-dimensional Peano continuum which is homotopy equivalent to $X_{0}$. Let $g_{0}: X_{0} \rightarrow Z$ and $g_{1}: Z \rightarrow X_{0}$ be the homotopy equivalence. According to our construction we have $X_{h}^{w} \subseteq Z$, and $g_{0}$ and $g_{1}$ are the identity on $X_{h}^{w}$. Hence $Z_{h \circ i_{*} \circ g_{1 *}}^{w}=\left(X_{0}\right)_{h \circ i_{*}}^{w}=X_{h}^{w}$. Let $h_{0}=h \circ i_{*} \circ g_{1 *}$ and $Z=Z_{h_{0}}^{w} \cup \bigcup_{i} A_{i}$ where $A_{i}$ s are open arcs. We use $\tilde{h}_{0}$ for the extension on $Z$ defined just before Lemma 3.9. Let $c_{x}$ be the constant path $x$ and $q=\psi\left(c_{x}\right)$, where $\psi$ and $\xi$ are defined for $h_{0}$. For a loop $l$ with base point $x$ in $X$, using Lemma 3.3 we have

$$
\begin{aligned}
h([l]) & =h \circ i_{*}([r \circ l])=h \circ i_{*} \circ g_{1 *}\left(\left[g_{0} \circ r \circ l\right]\right)=\varphi_{q}\left(\left[\xi\left(g_{0} \circ r \circ l\right)\right]\right) \\
& =\varphi_{q}\left(\tilde{h}_{0_{*}}\left(\left[g_{0} \circ r \circ l\right]\right)\right)=\varphi_{q} \circ\left(\tilde{h}_{0} \circ g_{0} \circ r\right)_{*}([l]) .
\end{aligned}
$$

Now $\tilde{h}_{0} \circ g_{0} \circ r$ is the desired continuous map.

When $x \notin X_{h}^{w}$, we choose a path $p$ from $x_{0} \in X_{h}^{w}$ to $x$. Then we have $h \circ \varphi_{p}: \pi_{1}\left(X, x_{0}\right) \rightarrow \pi_{1}(Y, y)$. By the preceding we have a continuous map $f: X \rightarrow Y$ and a path $q$ from $f\left(x_{0}\right)$ to $y$ such that $h \circ \varphi_{p}=\varphi_{q} \circ f_{*}$. Let $l$ be a loop with base point $x$. Then

$$
\begin{aligned}
h([l]) & =h \circ \varphi_{p} \circ \varphi_{p^{-}}([l])=h \circ \varphi_{p}\left(\left[p l p^{-}\right]\right) \\
& =\varphi_{q} \circ f_{*}\left(\left[p l p^{-}\right]\right)=\varphi_{q}\left(\left[(f \circ p) f \circ l(f \circ p)^{-}\right]\right) \\
& =\varphi_{q} \circ \varphi_{(f \circ p)^{-}} \circ f_{*}([l])=\varphi_{(f \circ p)^{-} q} \circ f_{*}([l]),
\end{aligned}
$$

which completes our proof. 
Proof of Corollary 1.3. By Theorem 2.1 we may assume $X=X^{w} \cup \bigcup_{i} A_{i}$ where each $A_{i}$ is an open arc. If $X^{w}=\emptyset$, then we obtain the conclusion easily. We suppose that $x_{0} \in X^{w}$. Since $f_{*}$ is injective, we have $X^{w}=X_{f_{*}}^{w}$ and we can define $\left(f_{*}\right)^{\sim}$ on $X$ according to the definition just before Lemma 3.9 For $x \in X_{f_{*}}^{w},\left(f_{*}\right)^{\sim}(x)$ is the unique point determined by Lemma 3.1 and hence $\left(f_{*}\right)^{\sim}(x)=f(x)$. Let $\psi: P_{X_{f_{*}}^{w}, x_{0}}(X) \rightarrow R P_{y}(Y)$ and $\xi: P_{X_{f_{*}}^{w}}(X) \rightarrow$ $R P(Y)$ be defined as before Lemma 3.3. For $x \in A_{i}$, we have $\left(f_{*}^{*}\right)^{\sim}(x)=$ $\xi\left(a_{i}\right)\left(a_{i}^{-1}(x)\right)$. Let $p$ be a path from $A_{i}^{1}$ to $x_{0}$. Then $\xi\left(a_{i}\right) \sim \psi\left(a_{i} p\right) \psi(p)^{-}$. By the uniqueness of $[\psi(p)]$ according to Lemma 3.1. we have $[\psi(p)]=[f \circ p]$ and $\left[\psi\left(a_{i} p\right)\right]=\left[f \circ\left(a_{i} p\right)\right]$ by the same argument. Now,

$$
\xi\left(a_{i}\right) \sim \psi\left(a_{i} p\right) \psi(p)^{-} \sim\left(f \circ a_{i}\right)(f \circ p)(f \circ p)^{-} \sim f \circ a_{i}
$$

and so $\left(f_{*}\right)^{\sim}=\xi\left(a_{i}\right) \circ a_{i}^{-1}$ and the restriction of $f$ to $\overline{A_{i}}$ are homotopic. By Lemma 4.1, $f$ and $\left(f_{*}\right)^{\sim}$ are homotopic as in the proof of Theorem 1.1. Now Lemma 3.10 implies the conclusion.

REMARK 4.2. (1) Theorem 1.2 can most probably be proved in the case where $Y$ is a planar continuum using a method of C. Kent [13].

(2) For topologists who are not familiar with wild topology, it seems to be difficult to understand what ideas work in the proofs of this paper. Theorem 2.1 reduces Peano continua to simple ones. The idea of its proof is standard in continuum theory going back to [1]. More unfamiliar parts seem to be in Section 3, which extend [8]. In [8] we show that many things about wild algebraic topology can be reduced to the Hawaiian earring and how the homomorphic image of the fundamental group of the Hawaiian earring can detect a point in the space in question due to the noncommutative Specker phenomenon. This phenomenon goes back to G. Higman and is explained in [8, Remark 3.16(4)]. The Higman theorem is related to the fundamental group and the shape group of the Hawaiian earring. An application to topology of this theorem appeared in [6], which was used in [11]. A more apparent topological use can be seen in [7, Corollary 2.11], where it is shown that every endomorphism of the fundamental group of the Hawaiian earring is conjugate to the homomorphism induced from a continuous map. This is a prototype of Lemma 3.1 by which we define $\tilde{h}$ on $X_{h}^{w}$ for a homomorphism $h$ between the fundamental groups of one-dimensional Peano continua. In the present paper we have extended the domain of $\tilde{h}$ to the whole space $X$ using Theorem 2.1 and strengthening results in [8] according to the ideas there. In other published papers topological use of the noncommutative Specker phenomenon can be seen in [2] and [16], though it is used implicitly there.

Acknowledgements. The author thanks G. Conner, U. Karimov, K. Kawamura and D. Repovš for their interest in this subject. The author 
is grateful to the referee for his very careful reading of this paper and of $[8$ as well, and his suggestions and detecting errors. The author was supported by the Grant-in-Aid for Scientific research (C) of Japan No. 20540097.

\section{References}

[1] R. H. Bing, Partitioning continuous curves, Bull. Amer. Math. Soc. 58 (1952), 11011110 .

[2] J. W. Cannon and G. R. Conner, On the fundamental groups of one dimensional spaces, Topology Appl. 153 (2006), 2648-2672.

[3] G. R. Conner and K. Eda, Correction to: "Algebraic topology of Peano continua" and "Fundamental groups having the whole information of spaces", Topology Appl. 154 (2007), 771-773.

[4] M. L. Curtis and M. K. Fort, The fundamental group of one-dimensional spaces, Proc. Amer. Math. Soc. 10 (1959), 140-148.

[5] R. J. Daverman, Decompositions of Manifolds, Academic Press, 1986.

[6] K. Eda, Free $\sigma$-products and noncommutatively slender groups, J. Algebra 148 (1992), 243-263.

[7] - Free $\sigma$-products and fundamental groups of subspaces of the plane, Topology Appl. 84 (1998), 283-306.

[8] - The fundamental groups of one-dimensional spaces and spatial homomorphisms, ibid. 123 (2002), 479-505.

[9] —, Algebraic topology of Peano continua, ibid. 153 (2005), 213-226.

[10] K. Eda and K. Kawamura, The fundamental groups of one-dimensional spaces, ibid. 87 (1998), 163-172.

[11] - - - The singular homology of the Hawaiian earring, J. London Math. Soc. 62 (2000), 305-310.

[12] R. Engelking, General Topology, Heldermann, 1989.

[13] C. Kent, Homomorphisms into the fundamental group of one-dimensional and planar continua, Master Thesis, Brigham Young Univ., 2008.

[14] J. C. Mayer, L. G. Oversteegen, and E. D.Tymchatyn, The Menger curve characterization and extension of homeomorphisms of non-locally-separating closed subsets, Dissertationes Math. 252 (1986).

[15] M. Meilstrup, Classifying homotopy types of one-dimensional Peano continua, Master Thesis, Brigham Young Univ., 2005.

[16] A. Zastrow, Construction of an infinitely generated group that is not a free product of surface groups and abelian groups, but which acts freely on an $\mathbb{R}$-tree, Proc. Roy. Soc. Edinburgh Sect. A 128 (1998), 433-445.

Katsuya Eda

School of Science and Engineering

Waseda University

Tokyo 169-8555, Japan

E-mail: eda@logic.info.waseda.ac.jp 